\title{
Towards Spectral Imaging and Elemental Mapping at Atomic Resolution
}

\author{
I.M. Anderson
}

Metals \& Ceramics Div., Oak Ridge National Laboratory, PO Box 2008, Oak Ridge TN 37831-6064

Spectral imaging, where a full spectrum is acquired at each pixel of an image, provides a method for surveying the compositional or chemical variations within a specimen with the characteristic spatial and spectral resolutions of the chosen technique [1]. Considerable effort has been devoted during the past decade to the development of spectral imaging, including data acquisition packages, automated data analysis methods, and the development of spectral imaging techniques for a variety of analytical signals [1-4]. The purpose of the present paper is to consider the application of spectral imaging methods to atomic-resolution compositional analysis in the scanning transmission electron microscope (STEM) with energy-dispersive X-ray (EDX) or electron energy-loss (EELS) spectroscopies.

STEM-EDX and -EELS spectral imaging provide a means of achieving the analytical goal of the Transmission Electron Aberration-corrected Microscope (TEAM) development project, which seeks to leverage the breakthrough development of aberration-correcting electron optics to achieve atomicresolution imaging and analysis of nanomaterials in 3D and real time [5]. Aberration correction of the STEM probe-forming lens by itself removes a longstanding limitation on performing quantitative compositional analysis at atomic resolution, namely the low signal levels available for elementspecific spectral features, by significantly increasing the available incident flux in an atomic-scale probe. However, atomic-resolution microanalysis may be frustrated by another more fundamental limitation, namely damage to the specimen by the intense probe. The realization of the analytical goal of the TEAM project will be predicated upon devising a data acquisition paradigm that allows acquisition of sufficient signal without first altering or destroying the specimen. Spectral imaging methods can be used to mitigate beam damage by allowing the signal to be acquired while keeping the beam moving, with short dwell times and multiple equivalent exposures. For EDX spectral imaging, the Position-Tagged Spectrometry (PTS) paradigm [6,7] would allow the most extreme implementation of this strategy, where the effective dwell time could be reduced below that necessary to acquire a single X-ray in a given pixel; however, there is no equivalent at this time for EELS, where the dwell time per pixel would be limited by the read-out rate of the spectrometer.

In addition to mitigating beam damage, STEM spectral imaging methods provide a built-in means of appropriately registering the acquired analytical signal with a given atomic column to be analyzed. Such "drift correction" is often implemented in some form with spectral imaging acquisition packages in order to mitigate distortion or blurring of the acquired image due to relative movement of the specimen with respect to the electron beam, arising from mechanical instabilities of the specimen stage (specimen drift) and electrical or thermal instabilities of the electron optical lenses (probe drift). The requirements for appropriate data registration are significantly more stringent for the atomic-resolution analysis envisioned under TEAM. In addition to specimen and probe drift, instabilities due to vibrations and stray fields presently frustrate the routine acquisition of undistorted Z-contrast images, which can be acquired with exposure times orders of magnitude shorter than those that will be necessary for quantitative compositional analysis. Drift correction for atomic-resolution STEM spectral images can be achieved "on-the-fly" by correlating the acquired 
analytical signals with the readout of detectors for signals with much higher cross sections, such the high-angle annular dark-field (HAADF) detector that is used to form a Z-contrast image. Accurate registration of the acquired analytical signal over multiple exposures could be achieved as long as the scan rate is slow enough to permit the acquisition of individual Z-contrast images with sufficient signal-to-noise to identify the most strongly scattering atomic columns within a unit cell, and to allow correlation of individual images with an undistorted reference image. The reference image could possibly be composed from a sequence of individual images that are subject to random distortions arising from mechanical and beam instabilities. This correlation of a time sequence of Zcontrast images, a method analogous to spectral imaging but with the non-spatial coordinate measured in time rather than energy, could provide a paradigm for the in-situ performance goal of the TEAM project, the characterization of structural variations in real time.

The spectral imaging paradigm may also provide other significant advantages for quantitative analysis, by allowing spatially resolved spectral variations to be measured as the incident probe is moved relative to the position of the prominent atomic columns. For example, spectral imaging would allow detection of dilute interstitial atoms that may not be apparent in the Z-contrast image. Spectral imaging will also help to discriminate between the localized atomic-column-specific signal and the spectral background that exists because of intrinsic delocalization of soft inner shell ionizations (e.g., O-K) or excitation of nearby atomic columns, arising from the finite incident beam convergence angle ( $\sim 25 \mathrm{mrad}$ for a $\mathrm{C}_{3}$-corrected probe) or scattering of the probe. For STEM-EDX, this spectral variation may also help to identify spectral contributions from secondary excitation, such as fast secondary electrons.

In summary, spectral imaging provides a paradigm that mitigates some of the most severe challenges to achieving quantitative compositional analysis at atomic resolution. Nevertheless, significant technique development will be required to implement this paradigm for quantitative compositional analysis via either EDX or EELS spectral imaging [8].

References

[1] I.M. Anderson, Microsc. Microanal. 6 (Suppl. 2) (2000) 1048.

[2] I.M. Anderson and J. Bentley, Microsc. Microanal. 3 (Suppl. 2) (1997) 931; Mater. Res. Soc. Symp. Proc. 458 (1997) 81.

[3] P.G. Kotula et al., Microsc. Microanal. 9 (2003) 1.

[4] P.G. Kotula and M.R. Keenan, these proceedings.

[5] http://ncem.lbl.gov/team.htm

[6] R.B. Mott and J.J. Friel, J. Microsc. 193 (1999) 2.

[7] J.J. Friel et al., these proceedings.

[8] Research at the Oak Ridge National Laboratory SHaRE Collaborative Research Center was sponsored by the Division of Materials Sciences and Engineering, U.S. Department of Energy, under contract DE-AC05-00OR22725 with UT-Battelle, LLC. 